\title{
A Finite Element Analysis of a Crack Penetrating or Deflecting into an Interface in a Thin Laminate
}

\author{
S. Kao-Walter ${ }^{1 a}$, P. Ståhle ${ }^{2 b}$, S.H. Chen ${ }^{3 c}$ \\ ${ }^{1}$ Dept. of Mechanical Engineering, School of Engineering, Blekinge Institute of Technology, S-371 \\ 79, Karlskrona, Sweden. \\ ${ }^{2}$ Div. of Material Mechanics, Malmö University, Malmö, Sweden. \\ ${ }^{3}$ LNM, Institute of Mechanics, Chinese Academy of Sciences, Beijing, 100080, China. \\ a Sharon.kao-walter@bth.se, ${ }^{\text {b }}$ per.stahle@ts.mah.se, ${ }^{c}$ chenshaohua72@hotmail.com
}

Keywords: Interface, Crack tip driving force, Adhesion, Deflecting, Penetrating.

\begin{abstract}
The crack tip driving force of a crack growing from a pre-crack that is perpendicular to and terminating at an interface between two materials is investigated using a linear fracture mechanics theory. The analysis is performed both for a crack penetrating the interface, growing straight ahead, and for a crack deflecting into the interface. The results from finite element calculations are compared with asymptotic solutions for infinitesimally small crack extensions. The solution is found to be accurate even for fairly large amounts of crack growth. Further, by comparing the crack tip driving force of the deflected crack with that of the penetrating crack, it is shown how to control the path of the crack by choosing the adhesion of the interface relative to the material toughness.
\end{abstract}

\section{Introduction}

Thin aluminium foil and polymer laminate composites have proved their usefulness and high potential through many applications in various fields. In particular for packaging materials, components made from laminated materials are frequently designed to withstand extreme loads, not the least during the production. A better understanding of the failure mechanisms is useful for the design process and the production process of laminate with these components. An important task in the present work is to study in detail the fracture behavior of a crack situated in an aluminium foil (Al-foil) layer and terminating at the interface between this and a low density polyethylene (LDPE) laminate.

Many investigations of the interaction between a crack and an interface have been performed. One of the earliest works on the subject was made by Zak and Williams [1]. Later, Wang and Ståhle [2] used a dislocation simulation to extend the analysis to finite cracks and, recently, Chen et al. [3] have studied a similar problem for a finite body. By applying a finite element method, [4-6] presented different analyses of stress intensity factors and energy release rates for cracks in different bi-materials.

The present work applies a finite element method to analyze the crack tip driving force of a precrack that penetrates or deflects to follow the interface between a stiff and a weak material. The material properties of an Al-foil/LDPE laminate have been selected because of its industrial importance. It is assumed that the pre-crack is located in the stiffer material and that the interface is a perfect bond between the two materials. Both materials are assumed to be the homogeneous.

\section{Basic Equations}

According to Zak and Williams [1], the stress distribution in the vicinity of a tip of a crack that is perpendicular to a bi-material interface can be written:

$$
\sigma_{i j}^{o}=\xi_{0} \sigma_{o}\left(a_{o} / r\right)^{\lambda_{o}} f_{i j}(\theta, \alpha, \beta)
$$


Here, $\sigma_{0}$ is a remotely applied stress, $\xi_{0}$ is a non-dimensional geometry dependent coefficient and $\lambda_{0}$ is obtained as the largest root in the interval $0 \leq \lambda_{0}<1$ of the following generating equation:

$$
\cos \lambda_{o} \pi=\frac{2(\beta-\alpha)}{1+\beta}\left(1-\lambda_{o}\right)^{2}+\frac{\alpha+\beta^{2}}{1-\beta^{2}} .
$$

The angular functions $f_{i j}$ are known (cf. [1]). Above, $\alpha$ and $\beta$ are the two so called Dundurs' parameters [7]:

$$
\alpha=\frac{\Gamma\left(\kappa_{2}+1\right)-\left(\kappa_{1}+1\right)}{\Gamma\left(\kappa_{2}+1\right)+\left(\kappa_{1}+1\right)}, \beta=\frac{\Gamma\left(\kappa_{2}-1\right)-\left(\kappa_{1}-1\right)}{\Gamma\left(\kappa_{2}+1\right)+\left(\kappa_{1}+1\right)},
$$

where $\Gamma=\mu_{1} / \mu_{2}, \kappa_{i}=3-4 v_{i}$ for the plane strain case and $\kappa_{i}=\left(3-v_{i}\right) /\left(1+v_{i}\right)$ for the plane stress case. The material parameters $v_{i}$ and $\mu_{i}$ are Poisson's ratio and the shear modulus of layer $i$ where $i=1$ or 2 .

For $\mu_{1} / \mu_{2} \rightarrow 0$, the expanded result of Eq. 2 gives (cf. [1])

$$
\lambda_{o}=1-0.88 \sqrt{\mu_{1} / \mu_{2}} \text {. }
$$

According to [3], this is so even in a finite solid in the region of $0<r / a_{o}<1.0$ for $\sigma_{x}$ and $0<r / a_{o}<0.5$ for $\sigma_{y}$.

A deflecting crack, $a$, along the interface is, according to [8], surrounded by the stress field

$$
\sigma_{i j}=\eta_{1} \sigma_{\infty}^{o}(a / r)^{1 / 2+i \varepsilon} g_{i j}(\theta, \alpha, \beta),
$$

where $g_{i j}$ are known angular functions $\left(c f\right.$. [1]), $\sigma_{\infty}$ is a remotely applied stress and $\eta_{1}$ is a geometry dependent constant. If the crack propagates a short distance, $a$, along the interface, via a kink, the stress surrounding the tip of the kink should be the stress field given in Eq. 1. The stresses in the field of Eq. 1 therefore form a boundary layer that embedded the crack tip and the kinking crack. Thus the stresses $\sigma_{i j}^{o}$ in Eq. 1 replace the remote stresses $\sigma_{\infty}^{o}$ in Eq. 5. Thus, the result is readily obtained as:

$$
\sigma_{i j}=\xi_{1} \sigma_{o}\left(a_{o} / a\right)^{\lambda_{o}}(a / r)^{1 / 2+i \varepsilon} g_{i j}(\theta, \alpha, \beta) \text {. }
$$

Here a geometry dependent coefficient $\xi_{1}$ replaces the constants $\xi_{0}$ and $\eta_{1}$ and

$$
\varepsilon=\frac{1}{2} \ln \left(\frac{1+\beta}{1-\beta}\right) .
$$

Eq. 6 is valid as long as the kink is fully embedded by the stress field related to Eq. 1 .

Similarly, the tip of a penetrating crack with the length, $a$, extending into the second material is surrounded by a stress field

$$
\sigma_{i j}=\eta_{2} \sigma_{\infty}(a / r)^{1 / 2} h_{i j}(\theta),
$$

where $\eta_{2}$ is an introduced geometry dependent constant. The angular functions $h_{\mathrm{ij}}$ are known as the angular functions of the Williams expansion. After replacing the stress $\sigma_{\infty}$ in Eq. 8 with $\sigma_{i j}^{o}$ defined in Eq. 1, the following expression is obtained for a penetrating crack embedded by the field related to Eq. 1.

$$
\sigma_{i j}=\xi_{2} \sigma_{o}\left(a_{o} / a\right)^{\lambda_{o}}(a / r)^{1 / 2} h_{i j}(\theta)
$$


Here $\xi_{2}$ is a constant. By Eq. 6 and Eq. 9, the crack tip driving force, $G$, may be written as follows (cf. [8]):

$$
G=\frac{1-v_{1}}{2 \mu_{1}} \xi \sigma_{o}^{2} a_{o}^{2 \lambda_{0}} a^{1-2 \lambda_{0}} .
$$

Here, $\xi$ is a constant related to Dunders' parameters. In the case when the crack deflects a distance $a$ into the interface $\xi=\xi_{1}(\alpha, \beta)$. While in the case when the crack penetrates a length $a$ into a homogenous material $\xi=\xi_{2}, c f$. [8].

One can also relate the crack tip driving force to the elastic energy release rate through [9]:

$$
G=\frac{\Delta}{2 B} \frac{d P}{d a}
$$

Here $\Delta$ is an applied constant displacement imposed on the edges of the specimen and $B$ is assumed to be $1 \mathrm{~mm}$ for the present analysis. $P$ is the resulting reaction force. After integrating over the full growth of the kink along the interface or equally over the full growth of the straight crack, Eq. 11 can be written as:

$$
\int_{0}^{a} G d a^{\prime}=\int_{P_{o}}^{P} \frac{\Delta}{2} d P^{\prime}=\frac{\Delta}{2}\left(P-P_{o}\right) .
$$

Here the growth is in both cases considered as starting from the original crack with the length $a_{o}$.

By using Eq. 10, the left side of the Eq. 12 becomes:

$$
\int_{0}^{a} G d a^{\prime}=\frac{\left(1-v_{1}\right) \xi \sigma_{o}^{2} a_{o}^{2 \lambda_{o}}}{2 \mu_{1}} \frac{a^{2\left(1-\lambda_{o}\right)}}{2\left(1-\lambda_{o}\right)} .
$$

Hence, after using the relation of Eq. 10, we finally obtain,

$$
G=\frac{\left(1-\lambda_{o}\right)}{a} \Delta\left(P-P_{o}\right) .
$$

This result is now based on the assumption that the stress fields described by Eq. 6 or alternatively Eq. 9 are valid. With Eq. 14 we are now equipped with a tool for evaluation of the asymptotic fields with regard to their applicability for larger kinks and for cracks extending far into the second material. The limitation to the applicability of the asymptotic fields will reveal itself as a difference of $G$ calculated using Eq. 10 and Eq. 14 by inserting the calculation results with finite element method. 


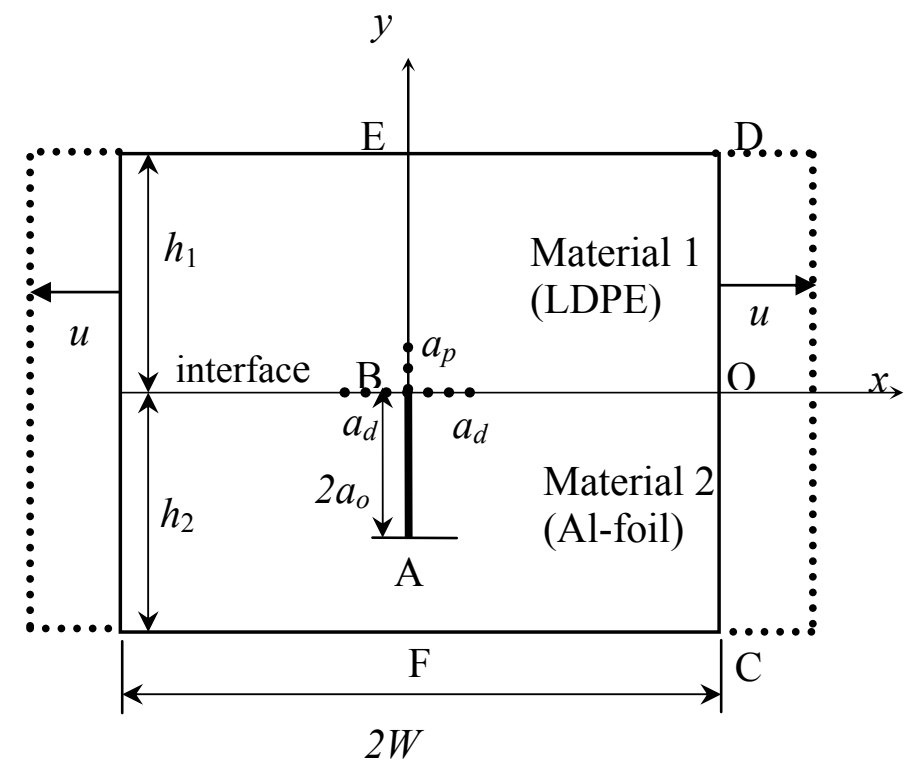

Fig. 1. A crack perpendicular to the bimaterial interface of a finite solid. Here, the penetrating crack and the double deflecting crack are illustrated by $a_{p}$ and $a_{d}$.

\section{Finite Element Analysis}

A general purpose finite element code [10] is used for the calculations. In total 7351 eight node plane elements with reduced integration is used. The ratio of the linear extent of largest versus the smallest element is 862 . Due to the symmetry, only half of the geometry is modelled.

A through thickness pre-crack with the length $a_{\mathrm{o}}$ is placed in the stiffer layer. The normalized geometry parameters are $W / a_{o}=32.0, h_{2} / a_{o}=2.0$ and $h_{1} / a_{o}=8$. A remote displacement $u_{o}$ was applied at the boundary DOC in Fig. 1. Dundurs' parameters where chosen to $\alpha=-0.992$ and $\beta=-$ 0.283 . For plan strain, this corresponds to, e.g., the shear modulus of Al-foil $\mu_{1}=52.5 \mathrm{MPa}$ and that of LDPE $\mu_{2}=13200 \mathrm{MPa}$. The corresponding Poisson's ratio $v$ is put 0.3 for both materials. Thus, the exponent $\lambda_{o} \approx 0.9444$ (cf. [6]).

In the case of penetration or deflection, a crack with the length $a_{p}$ or $a_{d}$ is placed as shown in Fig. 1. A series of reaction forces $P$ can be obtained. Thus, the crack tip driving force, $G_{p}$ or $G_{d}$, can be calculated for different crack lengths by applying Eq. 14 .

\section{Results and Discussions}

Fig. 2 shows the normalized crack tip driving force $G_{d} / G_{o}$ and $G_{p} / G_{o}$ for a pre-crack $a_{o}$ that penetrates or deflects a distance $a$ from the interface. It is assumed that the pre-crack $a_{o}$ is in the stiffer material and that the interface is perfectly bonded.

The Dundurs' parameter $\alpha=-0.992$ meaning that the stiffness of the stiffer material 2 is around 250 times larger than the stiffness of the weaker material 1 ( $c f$. Fig.1). Both the finite element results and the asymptotic results are shown in Fig. 2. The asymptotic solutions are found to be accurate even for a fairly large amount of crack growth.

Suppose now that the toughness of the interface is equal to Gic and the mode I toughness of the material is equal to Gc. As it has been discussed in [11], as long as the kink is fully embedded in the stress field Eq. 1 the crack will deflect into the interface and remain there if

$$
\frac{G_{i c}}{G_{c}}<\frac{G_{d}}{G_{p}}
$$



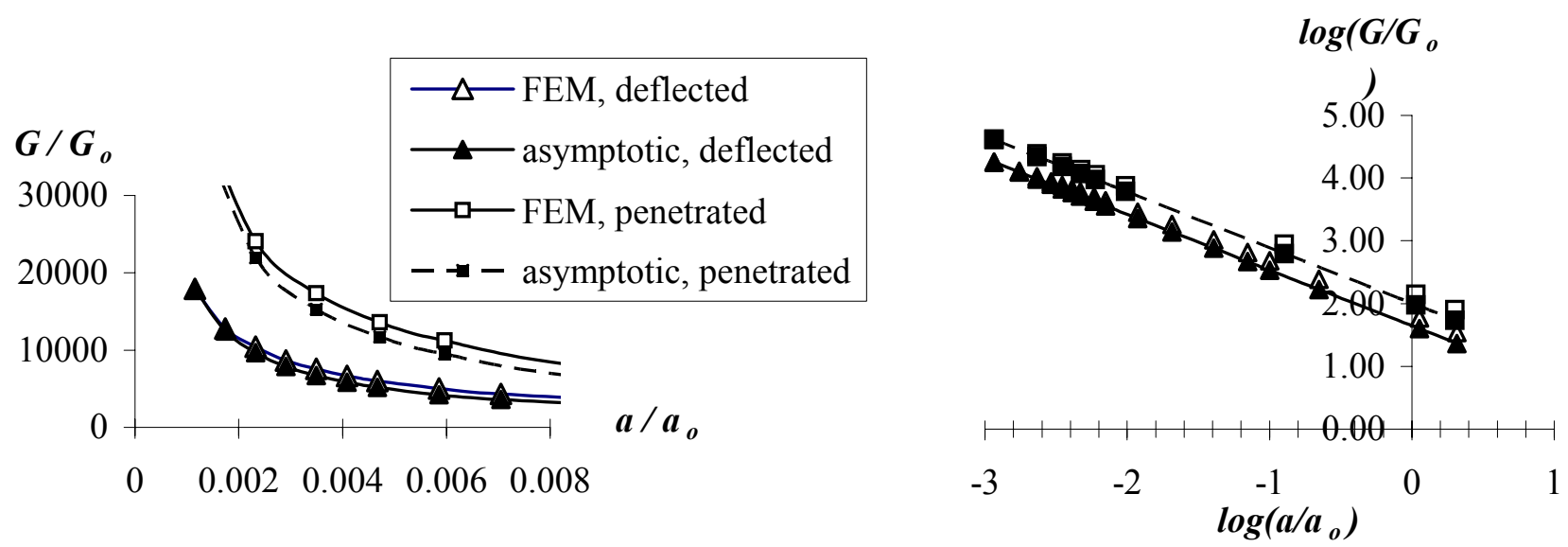

Fig. 2. Normalized crack tip driving force $G / G_{O}$ for deflected and penetrated crack versus normalized kink $a / a_{o}$. Here, $G_{o}=(\Delta / L)^{2} \mu_{2} h_{2}\left(1-\lambda_{o}\right)\left(1+v_{2}\right)$.

Fig. 3 shows the ratio of the crack tip driving force for deflection and for penetration, $G_{d} / G_{p}$, versus different $\alpha$ values. The results are compared to that from [8]. Good agreement can be found when $\alpha<0$. For $\alpha=-0.992$. This gives $G_{d} / G_{p}=0.488$ meaning that the crack tip driving force after deflection from the straight path is around half of the value for penetration (comparison is made at the same crack length). In reference [8] a different Dunders' parameter beta, i. e. $\beta=0$, was used. This may have caused the differences between the result of [8] and the present result (see Fig. 3). In the present analysis a ratio of length of kink to length of original crack $a / a o=0.0015$ which may, even though small, be insufficient to recover the asymptotic field surrounding the tip of a small kink. For $a=-0.992$ the result is backed up by the results in Fig. 2b. But for $a$ is larger than, say, 0.3 the result may be questioned.

This computation explains that the probability of crack deflection into the interface at increased displacement is large only if the toughness of the interface is less than half of the toughness of the weaker layer.

A full understanding of the final fracture of an Al-foil / LDPE laminate requires consideration of plastic deformation because of the high ductility of the LDPE layer. The toughness of a ductile material tested using a large-scale geometry cannot easily be transferred to a very small specimen.

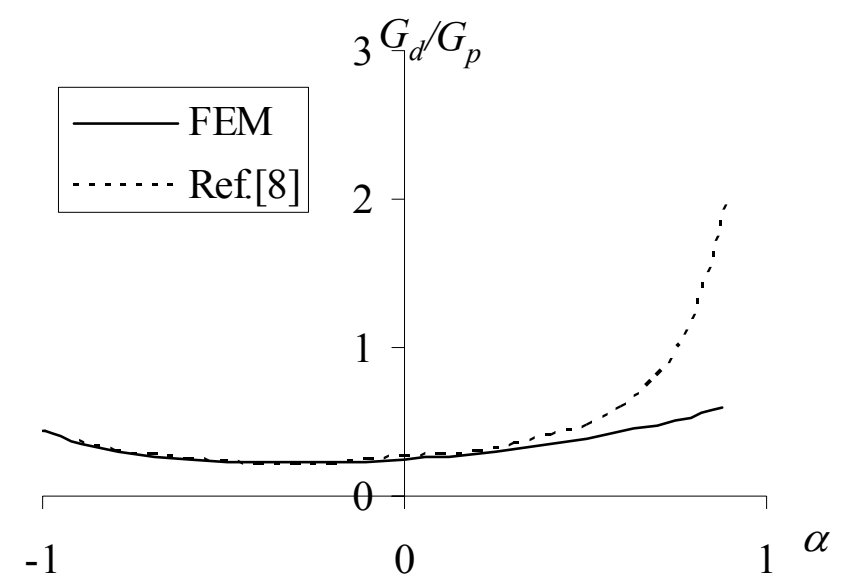

Fig. 3. The ratio of the crack tip driving force of a deflected crack to a double penetrated crack at the same crack length for different material combinations. 
Here, interaction with the Al-foil is expected since the linear extent of the plastic zone is of the same order of magnitude or larger than the thickness of the laminate layers (cf. [12]).

The crack tip driving force for a very long crack in the interface has a lower limit which equals the elastic energy stored in the two layers of the laminate. For a lower limiting toughness of the interface the crack will, therefore, just spontaneously grow in the interface at a given load.

\section{Conclusions}

The fracture of a layered laminate is studied as a cross-section crack propagation. A finite element method is used to calculate driving forces for the crack tip depending on whether the crack will grow into the interface or will just continue straight ahead.

The layer toughness should be confined between a lower limit and an upper limit to ensure delamination.

The results give guidance for the selection of the ratio of material stiffnesses and the ratio of fracture toughnesses of the undamaged layer and the interface to maximize the toughness of the entire structure. Here material properties of an Al-foil and an LDPE polymer laminate was chosen. For these materials, the conclusion is that the toughness of the interface should be at least half of the toughness of the LDPE layer if benefit from the toughening delamination is desired.Large-scale plastic effects were not considered in this analysis. It is anticipated that such effects would limit the reliability of the results for accurate quantitative predictions of ductile materials (e.g. Al-foil / LDPE).

\section{Acknowledgements}

This work was granted by Tetra Pak R\&D AB, Tetra Pak Carton Ambient AB and the Print Research Program (T2F). The authors would like to thank the computer- and program support at Blekinge Institute of Technology and FEM-tech AB. Thanks also to Mr. Chong Li at Malmö University for assisting during the early stages of the finite element calculation.

\section{References}

[1] A.R. Zak and M.L.Williams: J. Appl. Mech. Vol. 30 (1963), p. 142.

[2] T.C. Wang and P. Ståhle: Acta Mech. Sinica Vol-14 (1998), p. 27.

[3] S.H. Chen, T.C. Wang and S. Kao-Walter: Int. J. Solids and Structure Vol. 40 (2003), p. 2731.

[4] P. Ståhle, and C.F. Shih: Material Research Society Symposium Proceeding Vol.239 (1992), Boston.

[5] P. Delfin, J. Gunnars and P. Ståhle: Fatigue Fract. Engng Mater. Struct. Vol.18 (1995), p. 1201.

[6] S. Kao-Walter: On the Fracture of Thin Laminates (Doctoral thesis, Blekinge Inst. of Tech., Sweden, ISSN: 1650-2159, 2005)

[7] J. Dundurs: In Mathematics of Dislocation (edited by T. Mura, ASME, New York, 1969, p. 70.)

[8] M.Y. He, A. Evans and J.W. Hutchinson: Int. J. Solids and Structure Vol.31 (1994), p. 3443.

[9] T.L. Anderson: Fracture Mechanics, Fundamentals and Applications (CRC Press LLC, ISBN: 0-8493-4260-0, Florida, USA, 1995.)

[10] ABAQUS, User's manual, (version 6.4, Hibbit, Karlsson and Sorenson, ABAQUS, Inc. Printed in U.S.A., 2003.)

[11] M.Y. He and J.W. Hutchinson: Int. J. Solids and Structure Vol.25(1989), p. 1053. 